\title{
Complex quantum state generation and coherent control based on integrated frequency combs
}

\author{
Piotr Roztocki*, Stefania Sciara*, Christian Reimer*, Luis Romero Cortés, Yanbing Zhang, Benjamin \\ Wetzel, Mehedi Islam, Bennet Fischer, Alfonso Cino, Sai T. Chu, Brent E. Little, David J. Moss, \\ Lucia Caspani, José Azaña, Michael Kues, and Roberto Morandotti
}

\begin{abstract}
The investigation of integrated frequency comb sources characterized by equidistant spectral modes was initially driven by considerations towards classical applications, seeking a more practical and miniaturized way to generate stable broadband sources of light. Recently, in the context of scaling the complexity of optical quantum circuits, these on-chip approaches have provided a new framework to address the challenges associated with non-classical state generation and manipulation. For example, multi-photon and high-dimensional states were to date either inaccessible, lacked scalability, or were difficult to manipulate, requiring elaborate approaches. The emerging field of quantum frequency combs studying spectral multimode
\end{abstract}

Manuscript received July 1, 2018; revised Sept. 10, 2018; accepted XX XX, 2018. Date of publication XX XX, 2018; date of current version XX XX, 2018. This work was supported by the Natural Sciences and Engineering Research Council of Canada (NSERC) (Steacie, Strategic, Discovery, and Acceleration Grants Schemes, Vanier Canada Graduate Scholarships); MESI PSR-SIIRI Initiative; Canada Research Chair Program; Australian Research Council Discovery Projects (DP150104327); European Union's Horizon 2020 research and innovation program under the Marie Sklodowska-Curie grant (656607); CityU SRG-Fd program (7004189); Strategic Priority Research Program of the Chinese Academy of Sciences (XDB24030300); People Programme (Marie Curie Actions) of the European Union's FP7 Programme under REA grant agreement INCIPIT (PIOF-GA-2013-625466); Government of the Russian Federation through the ITMO Fellowship and Professorship Program (Grant 074-U 01); 1000 Talents Sichuan Program (China).

P. Roztocki, S. Sciara, and C. Reimer (* these authors contributed equally), L. Romero Cortés, Y. Zhang, B. Wetzel, M. Islam, B. Fischer, J. Azaña, M. Kues, R. Morandotti are with the INRS-EMT, Varennes, Québec, J3X 1S2, Canada (e-mail: piotr.roztocki@emt.inrs.ca; stefania.sciara@emt.inrs.ca; reimer@emt.inrs.ca; romero@emt.inrs.ca; yanbing.zhang@emt.inrs.ca; b.wetzel@sussex.ac.uk;

bennet.fischer@emt.inrs.ca; mehedi.islam@emt.inrs.ca; azana@emt.inrs.ca;

michael.kues@glasgow.ac.uk; morandotti@emt.inrs.ca).

S. Sciara and A. Cino are with the University of Palermo, Palermo, Italy (e-mail: alfonsocarmelo.cino@unipa.it).

C. Reimer is also with the John A. Paulson School of Engineering and Applied Sciences, Harvard University, Cambridge, 02138, USA.

B. Wetzel is also with the University of Sussex, Falmer, Brighton BN1 9RH, UK.

S.T. Chu is with the City University of Hong Kong, Tat Chee Avenue, Hong Kong, China (e-mail: saitchu@cityu.edu.hk).

B.E. Little is with the Chinese Academy of Science, Xi' an, China (e-mail: brent.little@opt.ac.cn).

D.J. Moss is with the Centre for Microphotonics, Swinburne University of Technology, Hawthorn, Victoria 3122, Australia (e-mail: dmoss@swin.edu.au).

L. Caspani is with the University of Strathclyde, Glasgow G1 1RD, UK (email: lucia.caspani@strath.ac.uk).

M. Kues is also with the University of Glasgow, Rankine Building, Oakfield Avenue, Glasgow G12 8LT, UK

R. Morandotti is also with the University of Electronic Science and Technology of China, Chengdu 610054, China and with ITMO Universiry, St Petersburg, Russia. sources based on the judicious excitation of (typically) thirdorder nonlinear optical micro-cavities, has begun to address these issues. Several quantum sources based on this concept have already been demonstrated, among them combs of correlated photons, cross-polarized photon pairs, entangled photon pairs, multi-photon states, and high-dimensional entangled states. While sources have achieved increasing complexity, so have coherent state processing operations, demonstrated in a practical manner using standard telecommunications components. Here, we review our recent work in the development of this framework, with a focus on multi-photon and high-dimensional states. The integrated frequency comb platform thus demonstrates significant potential for the development of meaningful quantum optical technologies.

Index Terms-Nanophotonics, Photonic integrated circuits, Quantum entanglement, Spontaneous emission.

\section{INTRODUCTION}

Oins OMPLEX optical quantum states are a key resource for investigations of fundamental questions in physics, as well as advanced applications such as quantum-based secure communications [1], powerful information processing schemes [2], and metrology/sensing [3]. Photon entanglement, i.e. the non-separability of a state despite even a physical (i.e. spatial) separation of its particles, is the prerequisite for several such uses. Concurrently, a state's complexity can be increased by raising the number $\mathrm{N}$ of photons and/or the photon dimensionality D [4], where the Hilbert space size scales as $\mathrm{D}^{\mathrm{N}}$. Furthermore, the exploitation of the resource provided by such states requires the enacting of specific, coherent transformations. Thus, realizing the full potential that photons offer for quantum technologies demands the custom preparation and coherent manipulation of large-scale quantum systems.

While the low-footprint, stability, and new functionalities offered by integrated photonics have made it a particularly attractive platform for quantum optics, the generation of complex photon states and their control have however remained largely inaccessible or have lacked scalability. In particular, the realization of multi-partite entangled states [5] is experimentally very challenging and has not been achieved with e.g. elaborate path-entanglement techniques. Likewise, using current approaches, the on-chip preparation of highdimensional (qudit) optical entanglement requires highly- 
complex and specialized components. Specifically, high-dimensional pathentanglement requires D coherently-excited identical sources and complicated circuits of beam-splitters [6], [7], whereas time-binentanglement demands elaborate and stable multi-arm interferometers [8].

Highlighted by the versatility and power of their recent exploitations in quantum optics, integrated frequency combs offer a unique framework for the generation and manipulation of complex states. Using both integrated optics and established telecommunications components, quantum frequency combs (QFCs) have recently

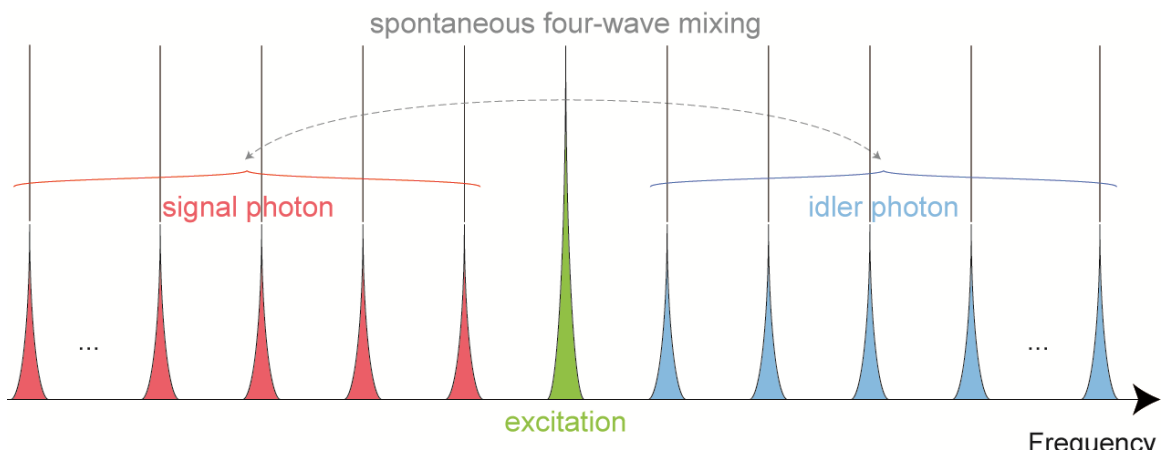

Fig. 1. Quantum frequency comb generation. An excitation field is coupled to the integrated nonlinear micro-cavity and is made, through filtering, to excite only a single resonance. Spontaneous four-wave mixing mediates the annihilation of two excitation field photons and the generation of a daughter photon pair (signal and idler) in a superposition of neighboring frequency modes. Adapted from [20].

been used to realize and coherently control the first on-chip sources of both multi-photon [9] and entangled highdimensional [10], [11] states. As such, they provide a promising framework for future quantum information technologies. In this article, we review our recent developments in this platform, with a focus on multi-photon and high-dimensional states.

\section{QUANTUM FREQUENCY COMBS}

Frequency combs are light sources with a broad spectrum of evenly-spaced frequency modes. The stabilization of classical comb sources effectively enabled a 'gear' connecting the optical and microwave frequency regimes, revolutionizing diverse fields such as spectroscopy and metrology, and earning their pioneers the 2005 Nobel Physics prize [12]. Yet, the challenges associated with bulk optical approaches for these devices have motivated investigations into more practical and portable platforms. On-chip nonlinear micro-cavities, with the optical excitation of one of their resonances, have been used as the main basis for integrated frequency comb sources [13]. The development of on-chip platforms for optical frequency comb applications is a very active and ongoing field, and has already yielded, e.g., mode-locked lasing [14], [15], CEO self-referenced combs [16], and much more.

However, when a nonlinear microcavity is excited below its optical parametric oscillation threshold (where the contribution of stimulated processes is negligible), two-photon states can be generated. In third-order nonlinear micro-resonators, spontaneous four-wave mixing (SFWM) can occur (Fig. 1), where two excitation field photons are

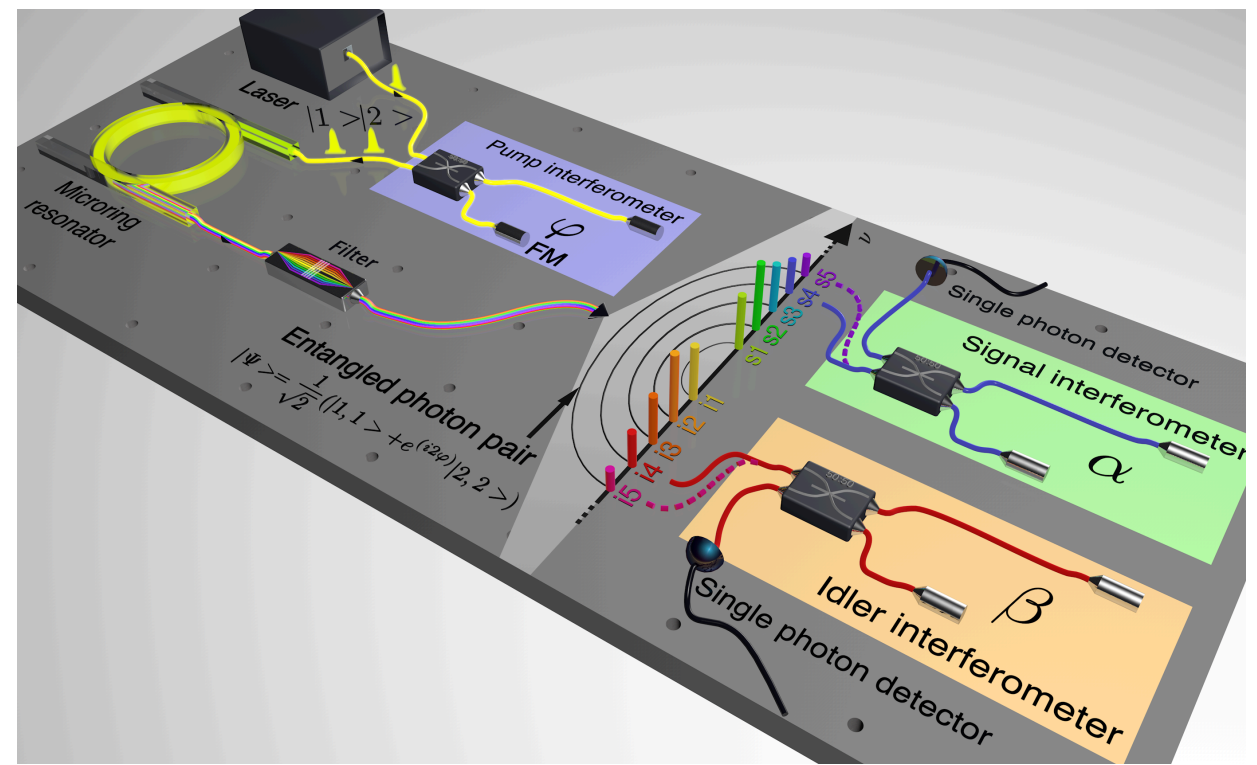

Fig. 2. Experimental setup for time-bin entangled photon generation. A pulsed laser is passed through an unbalanced fiber Michelson interferometer (composed of a 50:50 directional coupler and two Faraday Mirrors, FM), producing double pulses with a controlled phase difference $\varphi$. The pulses excite a single microring resonance, generating time-bin-entangled photon pairs on a frequency comb through SFWM. For state analysis, each photon of the spectrally-filtered photon pair is individually passed through an interferometer, with the temporal imbalance equal to the time-bin separation, and then detected with a single-photon detector. For four-photon measurements, four frequency modes symmetric to the excitation field are collected, passed through the interferometers, and spectrally-filtered prior to detection. Adapted from [9]. 
a

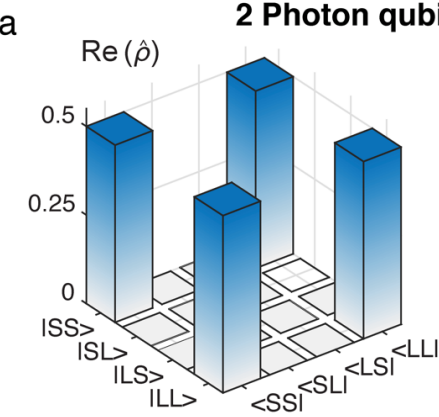

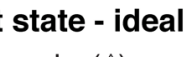

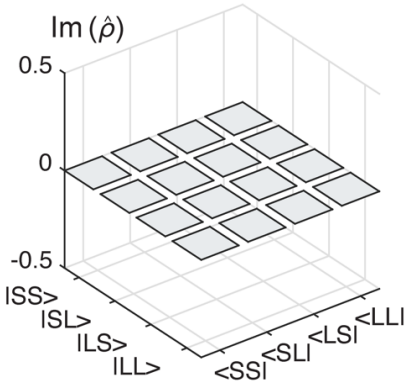

b
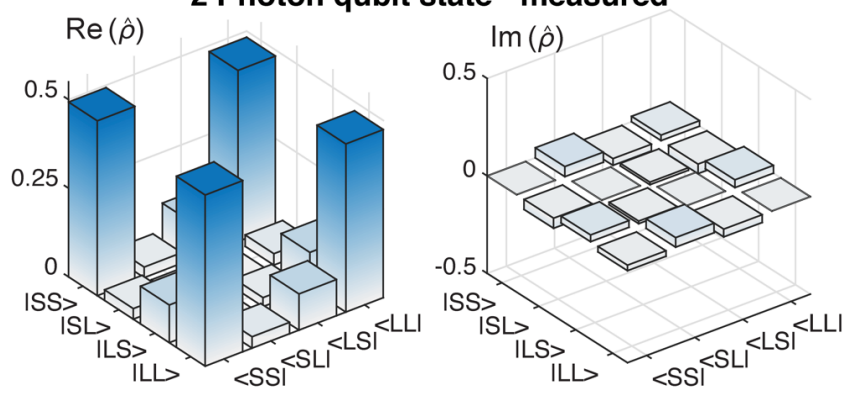
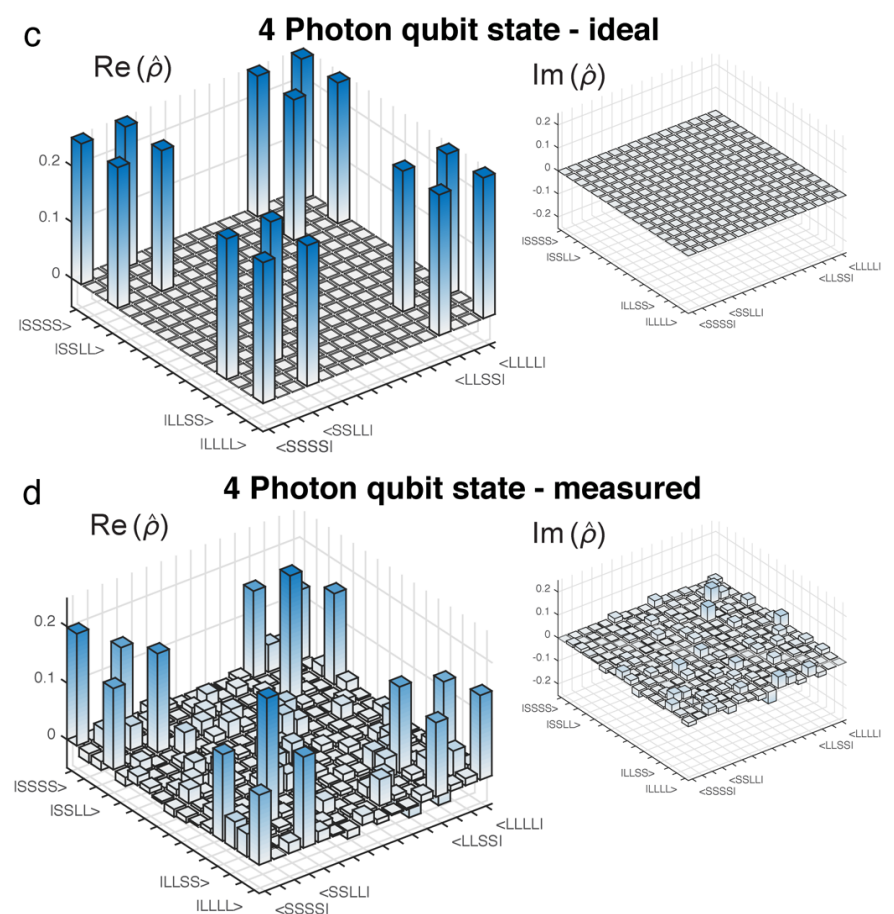

Fig. 3. Two- and four-photon qubit state tomography. The real (Re) and imaginary (Im) parts of the ideal density matrices of a two- and four-photon entangled qubit state are shown in (a) and (c) respectively, represented in the time-bin basis (|SS $\rangle,|\mathrm{SL}\rangle,|\mathrm{LS}\rangle,|\mathrm{LL}\rangle)$ and (|SSSS $\rangle,|\mathrm{SSSL}\rangle$, $<|L L L L\rangle)$. The measured density matrix of the two-photon state (b) agrees well with the ideal state, confirmed by a fidelity of $96 \%$. The measured density matrix of the four-photon entangled qubit state (d) reaches a fidelity of $64 \%$. Adapted from [9].

instabilities induced by thermal nonlinear effects [24]) and rather, pulsed excitation of such QFC micro-cavities -i.e., such that the photon pair generation still occurs probabilistically, but in discrete time windows- is particularly suitable for quantum information applications. Such pulsed sources significantly simplify the synchronization between photon sender and receiver stations, allow for the reduction of

detector noise counts through the use of temporal gating or post-selection, and can be used in systems that feature quantum repeaters or where an accurate knowledge of photon timing is required for two-photon interference [25]. Additionally, pulsed excitation stands at the basis of time-bin entanglement, a scheme that is very well suited for the existing fiber and electronics infrastructures [26], [27]. Moreover, pulsed excitation is necessary for the generation of fully separable two-photon states (i.e., exhibiting no spectral entanglement), enabling the heralding of pure, single frequency-mode photons (by detecting the other photon of the pair) [28]. This is of particular importance as pure single photon states are among the most fundamental entities in quantum optics, and are required for high-visibility multisource quantum interference (necessary for e.g. linear quantum optical computing) [2] as well as for scaling state complexity (towards, e.g., multi-photon states) [9]. Remarkably, the optical resonance intrinsically filters the spectral bandwidth of the excitation laser to match that of the generated photons, which removes the need for external filtering. Recent demonstrations of stable pulsed excitation schemes, besides focusing on power-efficiency, have also extended the concept to harmonic mode-locked excitation, with accompanying linear improvements in the photon pair generation rate [29].

Thanks to cavity field enhancement, micro-cavity-based sources can deliver high photon generation rates at low excitation powers - when compared to plain-waveguide sources [30]. As well, the small cavity footprint translates to resonance spacings (i.e. free spectral range, FSR) on the order of 10 's-100's GHz, allowing for frequency-mode addressability using standard telecommunications demultiplexers and spectral filters, as well as inter-mode mixing using off-the-shelf electro-optical modulators [10], [11]. Finally, photon emission at infrared wavelengths (we note emission of a broad comb over the S, C, L telecommunications bands) with narrow linewidths make these sources compatible with existing fiber and detection components, as well as future quantum memories.

Such quantum frequency comb source characteristics suggest their strong potential for non-classical technologies, and towards the scalable preparation and manipulation of complex states. Several multi-channel sources based on QFCs have thereby been demonstrated, among them combs of correlated photons [17], cross-polarized photon pairs [31], entangled photon pairs [9], [23], [32], multi-photon states [9], and frequency-bin entangled states [10], [11], [33].

\section{Multi-Photon Entangled States}

The multi-mode aspect of on-chip QFCs suggests an approach to multiplexing the generation of several channels of entangled photons in only a single spatial mode. An integrated micro-ring cavity (made of a high refractive index glass, Hydex) [34] resonance is excited with a pulse pair to demonstrate the realization of time-bin entangled photon pairs 


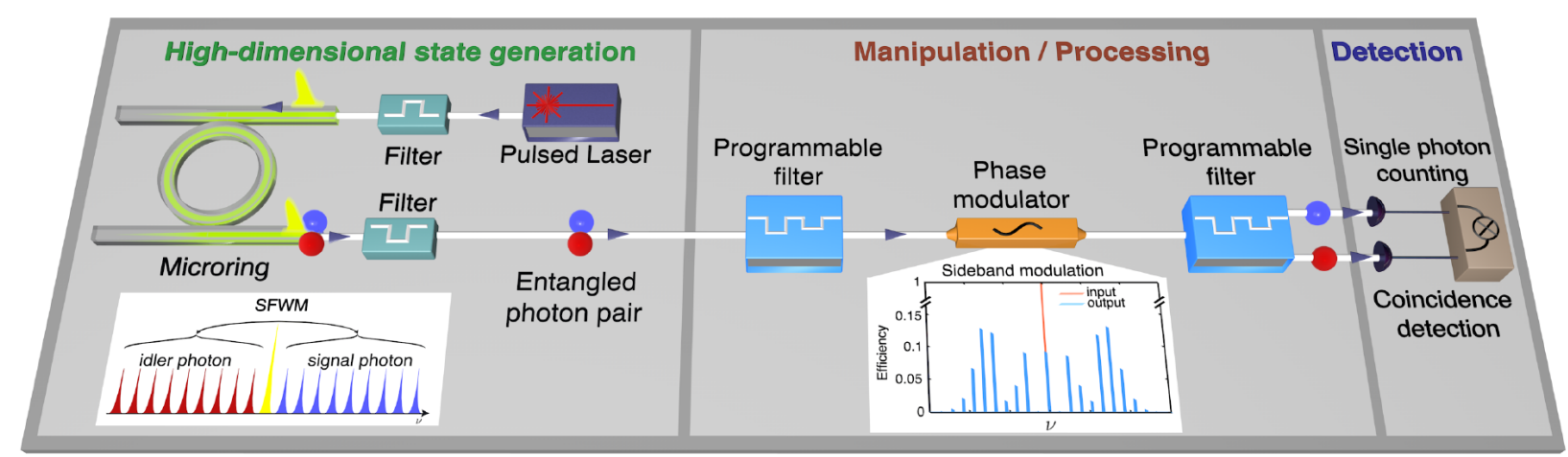

Fig. 4. Experimental setup for high-dimensional entangled state generation. A passively mode-locked laser was coupled into the integrated micro-ring resonator after being spectrally filtered to excite only a single resonance. Spontaneous four-wave mixing (see left inset and Fig 1.) led to the generation of photon pairs (signal and idler) spectrally symmetric to the excitation and in a quantum superposition of the frequency modes defined by the resonances. Programmable filters and a modulator were used for manipulating the state (the right inset shows frequency sideband generation by the modulator as a function of frequency $v$ ), before the signal and idler photons were detected by two single photon counters.

Adapted from [10].

(with generation rates of around $300 \mathrm{kHz}$ per channel) [27] over the entire frequency comb spectrum (where the daughter photon pair is produced in a superposition of two discrete generation times, corresponding to the two coherent-butseparate pulsed excitations of the micro-ring). Stabilized fiber interferometers, comprised of standard fiber telecommunications elements, are used to prepare the pulse pair and project the time-bin entangled qubits for state processing (Fig. 2). This setup enabled the measurement of the quantum interference between the signal and idler photons. An entangled system can be fully characterized by means of quantum state tomography, which allows to experimentally reconstruct the state density matrix, thus providing a complete description of the system. Furthermore, quantum interference can be measured to verify if the generated quantum state violates a Bell inequality, thus confirming its non-local properties. We selected five different frequency channel pairs within the $\mathrm{C}$ band and recorded quantum interference with raw visibilities above $82.4 \%$, which, being greater than $71 \%$, confirm non-locality and entanglement through the violation of the Clauser-Horne-Shimony-Holt (Bell-like) inequality [35]. After subtracting the measured background, the visibility was found to be above $93.2 \%$ for all channel pairs. We used the same setup to enact state density matrix reconstruction measurements. The retrieved density matrix of the two-photon state agrees very well with the expected ideal state, confirmed by a measured fidelity of $96 \%$ (Fig. 3).

Finally, multi-photon states have been demonstrated using e.g. bulk platforms [36], but never on a portable integrated platform. The distinctive multimode characteristic of the frequency comb architecture can be extended to easily create multi-photon entangled quantum states. In the comb, signalidler pairs may be generated on, e.g. two separate resonance pairs simultaneously. Here, the post-selection of these events enables the generation of a four-photon time-bin entangled state. We confirm the realization of this four-photon entangled state through four-photon quantum interference (with fourphoton detection rates of around $200 \mathrm{mHz}$ ), from which we extracted a measured visibility of $89 \%$ without background correction [9]. Furthermore, we repeated the four-photon measurement by selecting different combinations of four modes, always observing four-photon entanglement. The measured density matrix of the state reaches a fidelity of $64 \%$ (Fig. 3), comparable in quality to other four-photon states generated via non-integrated approaches.

\section{High-Dimensional Entangled States}

In order to generate high dimensional entangled states, we excited a single resonance of an integrated micro-ring resonator with a spectrally-filtered mode-locked laser [10]. Due to the broad phase-matching condition, the generated photon pairs cover multiple resonances (with generation rates of around $500 \mathrm{kHz}$ ). Furthermore, the photons are intrinsically created as a high-dimensional superposition of resonance frequency mode states, and are entangled due to SFWM energy conservation. The exploitation of such highdimensional states for quantum information processing motivates the need for high-dimensional operations that enable access to multiple modes with a minimum number of components. We thus employed a concatenation of standard telecommunications components for the coherent manipulation of the state within a single spatial mode, using such elements as programmable spectral phase filters and electro-optical phase modulators (Fig. 4). The programmable filters are used to impose an arbitrary phase and amplitude mask on the frequency components of the quantum state, while phase modulation deterministically shifts and mixes the different frequency components in a designed manner (Fig. 5).

The control scheme can then be used to implement arbitrary phase gates, as well as to perform deterministic highdimensional projection measurements. We first use these components to characterize the dimensionality of the state, and conclude, using joint spectral intensity [37] and second-order coherence function [38] measurements, that the quantum state has a Schmidt number of 10 and Hilbert space dimensionality of 100 , which is formed by two entangled qudits with $D=10$. We then validate our platform by measuring violations of a high-dimensional Bell inequality and performing quantum state tomography (Fig. 6). Specifically, we measure quantum interference for $\mathrm{D}=2,3$, and 4 , where we measure visibilities of $83.7 \%, 86.6 \%$, and $86.4 \%$, all violating their high- 


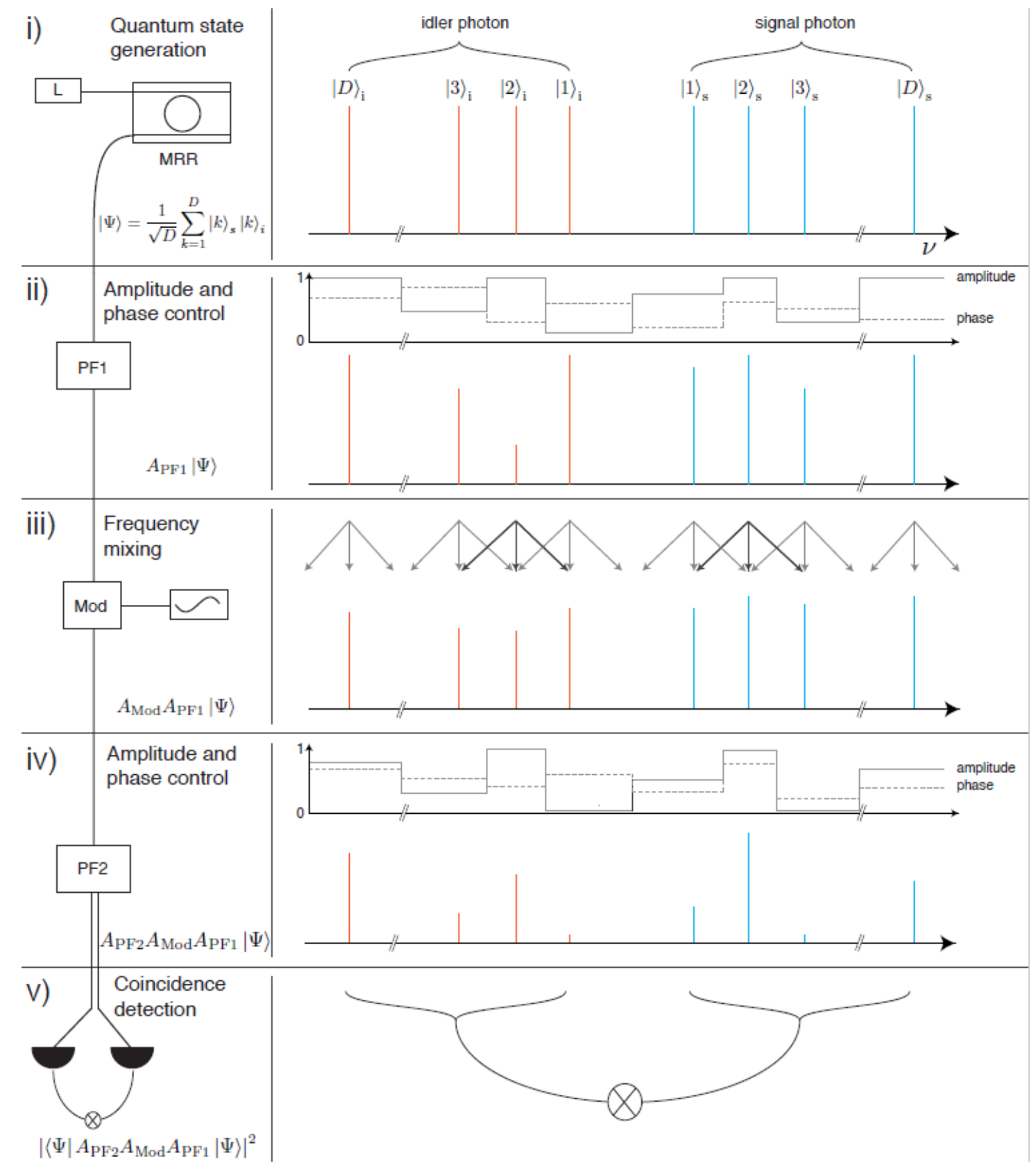

Fig. 5. Schematic of the coherent control scheme for high-dimensional frequency-bin entangled states. Individual steps to control, manipulate and characterize the quantum states are displayed, showing for each one the equipment used and a schematic of the modification imposed on the quantum state in the spectral domain. i, The initial states $|\Psi\rangle$ were generated using the micro-ring resonator (MRR, see Fig. 4). ii, Using a programmable filter (PF1), any arbitrary spectral phase and amplitude mask can be imposed on the quantum states for manipulation. iii, An electro-optic modulator (Mod) driven by a radio-frequency synthesizer was used to coherently mix different frequency components of the high-dimensional states. iv, A second programmable filter (PF2) can impose an amplitude and phase mask and route the signal and idler to two different paths. $v$, The photons were then detected using single photon counters and timing electronics. Adapted from [10].

dimensional Bell inequalities for $\mathrm{D}=2,3$, and 4 , respectively [39]. We then performed quantum state tomography to extract the state density matrices also for $\mathrm{D}=2,3$, and 4 , confirming that the experimental quantum states are very close to the ideal maximally entangled states with measured fidelities of $88.5 \%$, $80.9 \%$, and $76.6 \%$ for $\mathrm{D}=2,3$, and 4 , respectively [10]. Furthermore, by sending the entangled states through a 24.2km-long fiber telecommunications system (standard single mode and dispersion-compensating fiber elements), and then repeating the Bell inequality test, we confirmed that the entanglement was preserved over large propagation distances.

\section{CONCLUSION}

While the investigation of integrated frequency combs was originally motivated by classical applications, they have further been shown to be a scalable and versatile platform for quantum state generation and a practical potential platform for optical quantum information processing. Recent work has demonstrated the extension of these concepts towards e.g. a 
a

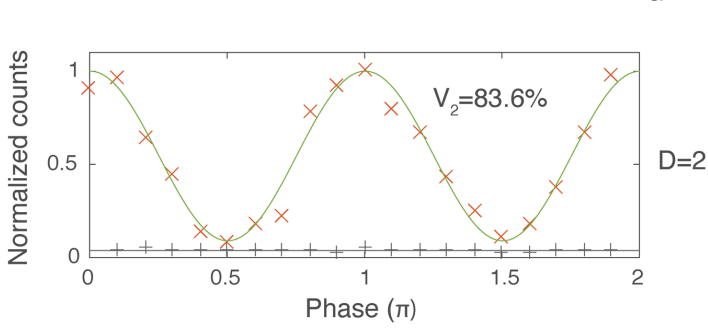

b

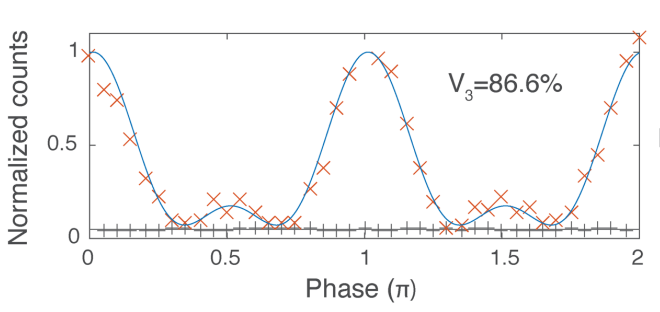

C

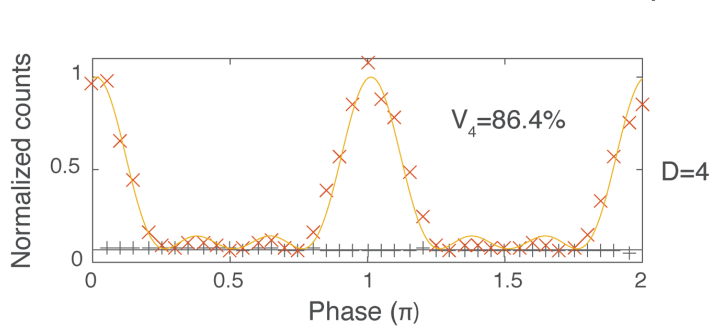

f d
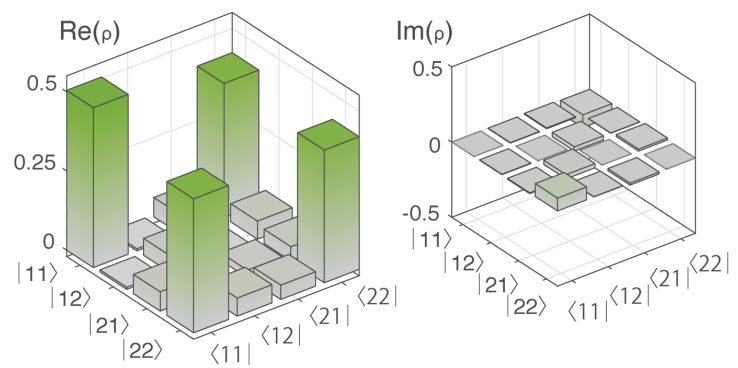

e
Reconstructed density matrix
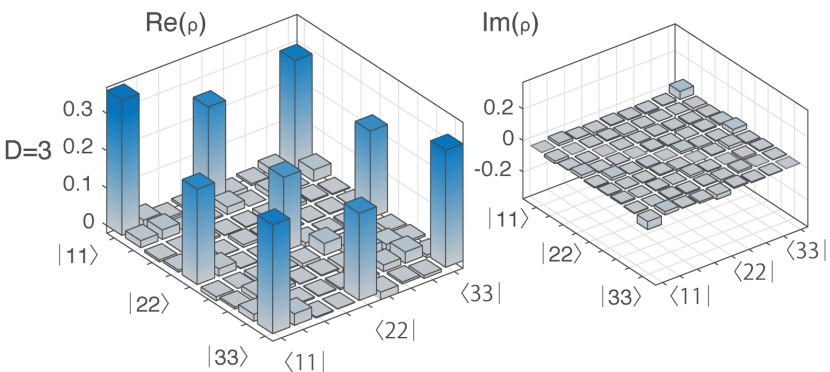

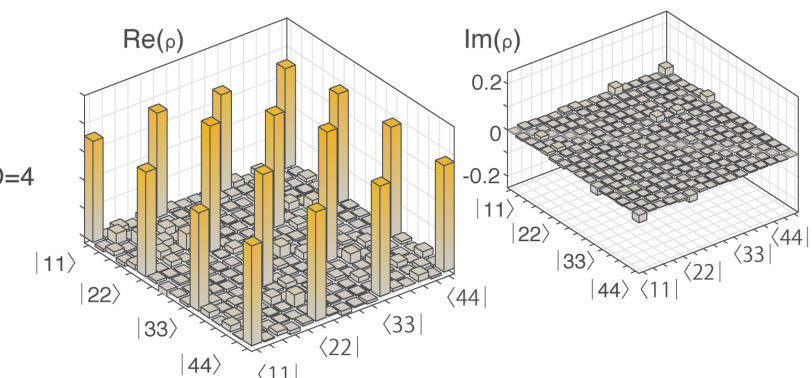

Fig. 6. Characterization of generated high-dimensional entangled states. For the quantum interference characterization of qudits with $\mathrm{D}=2(\mathrm{a}), \mathrm{D}=3$ (b) and $\mathrm{D}=4$ (c), the states were projected on a superposition of $\mathrm{D}$ frequency modes with different phases. By changing these phases, a variation in the coincidence counts was measured (the flat black curve being the recorded background). Raw visibilities of $83.6 \%, 86.6 \%$ and $86.4 \%$ for the quantum interference were obtained without background subtraction, exceeding the visibilities of $71 \%, 77 \%$ and $81.7 \%$, that are respectively required to violate a Bell inequality for the $\mathrm{D}=2, \mathrm{D}=3$ and $\mathrm{D}=4$ states. By exploiting the ability to carry out arbitrary projection measurements on the signal and idler photon independently, we performed full quantum state tomography for the entangled qudit states. We achieved fidelities of $88.5 \%, 80.9 \%$, and $76.6 \%$ for $\mathrm{D}=2$ (d), $\mathrm{D}=3$ (e) and $\mathrm{D}=4$ (f), respectively, demonstrating very good agreement between the measured and the expected maximally entangled states. Adapted from [10].

comb with $50 \mathrm{GHz}$ resonance spacing [11], an exciting development as lower micro-cavity FSRs simultaneously increase addressable dimensionality and ease the task of coherent manipulation. Finally, microcavity-based complex states and their temporal and spectral manipulation via standard telecommunications components can, especially if combined with gate implementations presently under development [40]-[42], open up new venues for reaching the state complexities and processing capabilities required for meaningful quantum information science.

\section{REFERENCES}

[1] H. J. Kimble, “The quantum internet," Nature, vol. 453, no. 7198, pp. 1023-1030, Jun. 2008.

[2] E. Knill, R. Laflamme, and G. J. Milburn, "A scheme for efficient quantum computation with linear optics," Nature, vol. 409, no. 6816, pp. 46-52, Jan. 2001.

[3] Y. Israel, S. Rosen, and Y. Silberberg, "Supersensitive polarization microscopy using NOON states of light," Phys. Rev. Lett., vol. 112, no. 10, p. 103604, Mar. 2014.

[4] Y. Zhang, F. S. Roux, T. Konrad, M. Agnew, J. Leach, and A. Forbes, "Engineering two-photon high-dimensional states through quantum interference," Sci. Adv., vol. 2, no. 2, pp. e1501165e1501165, Feb. 2016.

[5] H. J. Briegel and R. Raussendorf, "Persistent entanglement in arrays of interacting particles," Phys. Rev. Lett., vol. 86, no. 5, pp. 910913, Jan. 2001.

[6] C. Schaeff, R. Polster, R. Lapkiewicz, R. Fickler, S. Ramelow, and A. Zeilinger, "Scalable fiber integrated source for higherdimensional path-entangled photonic quNits," Opt. Express, vol. 20, no. 15 , p. 16145 , Jul. 2012.

[7] J. Wang et al., "Multidimensional quantum entanglement with large-scale integrated optics," Science, vol. 360, no. 6386, pp. 285291, Apr. 2018.

[8] R. T. Thew, A. Acin, H. Zbinden, and N. Gisin, "Experimental realization of entangled qutrits for quantum communication," Quantum Inf. Comput., vol. 4, no. 2, p. 093, Feb. 2004.

[9] C. Reimer et al., "Generation of multiphoton entangled quantum states by means of integrated frequency combs," Science, vol. 351, no. 6278, pp. 1176-1180, Mar. 2016.

[10] M. Kues et al., "On-chip generation of high-dimensional entangled quantum states and their coherent control," Nature, vol. 546, no. 7660, pp. 622-626, Jun. 2017.

[11] P. Imany et al., "50-GHz-spaced comb of high-dimensional frequency-bin entangled photons from an on-chip silicon nitride microresonator," Opt. Express, vol. 26, no. 2, p. 1825, Jan. 2018. "The Nobel Prize in Physics 2005." [Online]. Available: https://www.nobelprize.org/nobel_prizes/physics/laureates/2005/ind ex.html. [Accessed: 15-Jun-2018]. 
[13] P. Del'Haye, A. Schliesser, O. Arcizet, T. Wilken, R. Holzwarth, and T. J. Kippenberg, "Optical frequency comb generation from a monolithic microresonator," Nature, vol. 450, no. 7173, pp. 12141217, Dec. 2007.

[14] M. Peccianti et al., "Demonstration of a stable ultrafast laser based on a nonlinear microcavity," Nat. Commun., vol. 3, p. 765, Jan. 2012.

[15] K. Saha et al., "Modelocking and femtosecond pulse generation in chip-based frequency combs," Opt. Express, vol. 21, no. 1, p. 1335, Jan. 2013.

[16] V. Brasch et al., "Photonic chip-based optical frequency comb using soliton Cherenkov radiation," Science, vol. 351, no. 6271, pp. 357360, Jan. 2016.

[17] C. Reimer et al., "Integrated frequency comb source of heralded single photons," Opt. Express, vol. 22, no. 6, p. 6535, Mar. 2014.

[18] L. Caspani et al., "Multifrequency sources of quantum correlated photon pairs on-chip: a path toward integrated quantum frequency combs," Nanophotonics, vol. 5, no. 2, pp. 351-362, Jan. 2016.

[19] L. Caspani et al., "Integrated sources of photon quantum states based on nonlinear optics," Light Sci. Appl., vol. 6, no. 11, p. e17100, Nov. 2017.

[20] P. Roztocki et al., "Integrated generation of complex optical quantum states and their coherent control," in Nanophotonics Australasia 2017, 2018, p. 39.

[21] R. W. Boyd, Nonlinear optics, 3rd ed. Elsevier, 2008.

[22] E. Engin et al., "Photon pair generation in a silicon micro-ring resonator with reverse bias enhancement," Opt. Express, vol. 21, no. 23, pp. 27826-27834, Nov. 2013.

[23] D. Grassani et al., "Micrometer-scale integrated silicon source of time-energy entangled photons," Optica, vol. 2, no. 2, p. 88, Feb. 2015.

[24] T. Carmon, L. Yang, and K. J. Vahala, "Dynamical thermal behavior and thermal self-stability of microcavities," Opt. Express, vol. 12, no. 20, p. 4742, Oct. 2004.

[25] S. Takeuchi, "Recent progress in single-photon and entangledphoton generation and applications," Jpn. J. Appl. Phys., vol. 53, no. 3, p. 030101, Mar. 2014.

[26] T. Pittman, "It's a good time for time-bin qubits," Physics (College. Park. Md)., vol. 6, p. 110, Oct. 2013.

[27] J. Brendel, N. Gisin, W. Tittel, and H. Zbinden, "Pulsed energy-time entangled twin-photon source for quantum communication," Phys. Rev. Lett., vol. 82, no. 12, pp. 2594-2597, Mar. 1999.

[28] L. G. Helt, Z. Yang, M. Liscidini, and J. E. Sipe, "Spontaneous four-wave mixing in microring resonators," Opt. Lett., vol. 35, no. 18, p. 3006, Sep. 2010.

[29] P. Roztocki et al., "Practical system for the generation of pulsed quantum frequency combs," Opt. Express, vol. 25, no. 16, p. 18940 , Aug. 2017.

[30] D. Bonneau, J. W. Silverstone, and M. G. Thompson, "Silicon quantum photonics," in Silicon Photonics III, vol. 122, L. Pavesi and D. J. Lockwood, Eds. Berlin, Heidelberg: Springer Berlin Heidelberg, 2016, pp. 41-82.

[31] C. Reimer et al., "Cross-polarized photon-pair generation and bichromatically pumped optical parametric oscillation on a chip," Nat. Commun., vol. 6, no. 1, p. 8236, Dec. 2015.

[32] F. Mazeas et al., "High-quality photonic entanglement for wavelength-multiplexed quantum communication based on a silicon chip," Opt. Express, vol. 24, no. 25, p. 28731, Dec. 2016.

[33] D. Rieländer et al., "Frequency-bin entanglement of ultra-narrow band non-degenerate photon pairs," Quantum Sci. Technol., vol. 3, no. 1, p. 014007, Jan. 2018.

[34] D. J. Moss, R. Morandotti, A. L. Gaeta, and M. Lipson, "New CMOS-compatible platforms based on silicon nitride and Hydex for nonlinear optics," Nat. Photonics, vol. 7, no. 8, pp. 597-607, Jul. 2013.

[35] J. F. Clauser, M. A. Horne, A. Shimony, and R. A. Holt, "Proposed experiment to test local hidden-variable theories," Phys. Rev. Lett., vol. 23 , no. 15 , pp. 880-884, Oct. 1969.

[36] X.-L. Wang et al., "Experimental ten-photon entanglement," Phys. Rev. Lett., vol. 117, no. 21, p. 210502, Nov. 2016.

[37] C. K. Law, I. A. Walmsley, and J. H. Eberly, "Continuous frequency entanglement: effective finite Hilbert space and entropy control," Phys. Rev. Lett., vol. 84, no. 23, pp. 5304-5307, Jun. 2000.

[38] A. Christ, K. Laiho, A. Eckstein, K. N. Cassemiro, and C. Silberhorn, "Probing multimode squeezing with correlation functions," New J. Phys., vol. 13, no. 3, p. 033027, Mar. 2011.

[39] D. Collins, N. Gisin, N. Linden, S. Massar, and S. Popescu, "Bell inequalities for arbitrarily high-dimensional systems," Phys. Rev. Lett., vol. 88, no. 4, p. 040404, Jan. 2002.

[40] H.-H. Lu et al., "Electro-optic frequency beam splitters and tritters for high-fidelity photonic quantum information processing," Phys. Rev. Lett., vol. 120, no. 3, p. 030502, Jan. 2018.

[41] H.-H. Lu, J. M. Lukens, N. A. Peters, B. P. Williams, A. M. Weiner, and P. Lougovski, "Controllable two-photon interference with versatile quantum frequency processor," arXiv Prepr. arXiv1803.10712, Mar. 2018.

[42] P. Imany et al., "Deterministic optical quantum logic with multiple high-dimensional degrees of freedom in a single photon," arXiv Prepr. arXiv1805.04410, May 2018. 